ISSN 1991- 8690

website :http:// jsci.utq.edu.iq
الترقيم الدولي • 1991 ـ ـ 19.

Email: utjsci@utq.edu.iq

\title{
تأثثربعض المستخلصات النباتية المائية والمركبات الكيمياوية المثبطة لأنزيم اليوريز في خفض تطاير الامونيا من اليوريا المضافه حقليا لترب اهوار ذي قار
}

\author{
رزاق غازي نغيمش \\ عبد المهدي صالح الأنصاري \\ جامعة البصرة كلية الزراعة - قسم التربة والمباه \\ *جامعة ذي قار كلية العلوم - قسم علوم الحياة
}

المستخانص

أجريت الدراسة لبيان تأثير معاملة اليوريا بالمستخلصات النباتيه المائية والمركبات الكيمياوية الهيدروكينون(HQ) وثايوكبريتات الامونيوم) و

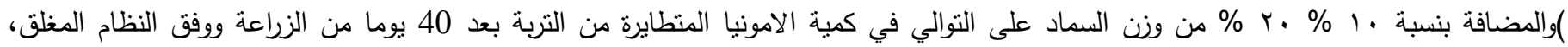

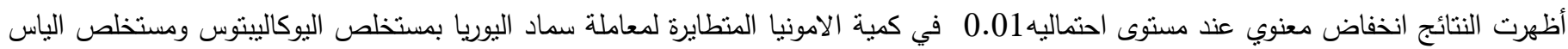
ومستخلص ليف النخيل ومستخلص قش الرز ومستخلص جذور الثيل ومركب HQ و 30.320 ATS و 32.045 و 26.761 و 29.643 و

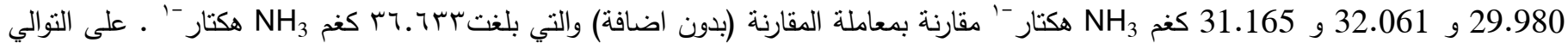
اختلفت معاملات المستخلصات النباتية المائية معنويا وبمستوى 0.01 فيما بينها كما تفوقت معاملات المستخلصات النباتية المائية في خفض كمية الامونيا المتطايرة معنويا وبمستوى احتمالي 0.01 عن معاملات HQ و ATS باستثناء معاملة مستخلص الياس الذي لم يختلف معنويا عن معاملة

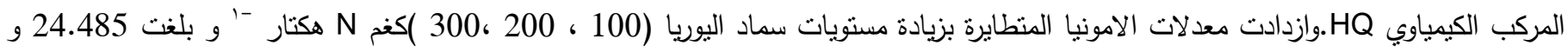

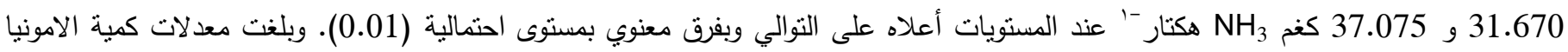

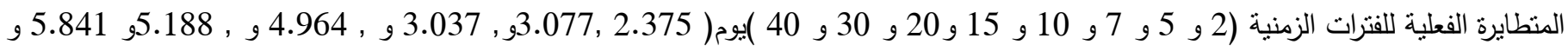

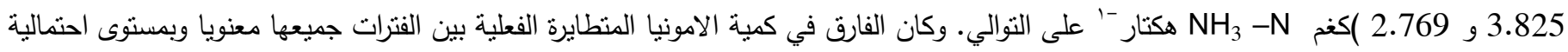

\section{Abstract}

The present study was inducted to show the effect of aqueous plant extracts and chemical compounds thiosulphate ammonium (ATS) and hydroqenone (HQ)upon Ammonia volatilization which added 10\% .20\% respaicvtily from urea weight after 40 days of fertilizer adding accoding to close system. The results were appeared significantly reduction at 0.01 level volatilization ammonia quantity for urea treated with the extract of Eucalyptus camaldulensrs Dehn L. , Myrtus communis L, Phoenix dactlifera L, Oryza sativa, Cynodon dactylon L.Pers HQ and ATS30.320, $32.045,26.761,29.643,29.980,32.061$ and $31.165 \mathrm{Kg} \mathrm{NH}_{3} \mathrm{Ha}^{-1}$ compared with the control treatment that was $36.633 \mathrm{~kg}$ NH3 Ha-1.All treatments of the plant extracts were significantly difference with (HQ and ATS) treatments and with themselves at level probably (0.01) except MYrtus communis L. treatment was not significantly difference with HQ treatment extract, An amount of ammonia volatilization increased significantly when increased the levels of Nitrogen $(100,200$, and 300)kg N Ha-1 were $(24.485$, 31.670, and 37.075) kg NH3 Ha-1 to all levels respectively . The rates for active amount of ammonia volatilization to all time periods $(2,5,7,10,15,20,30$,and 40)days were $(2.375,3.077,3.37,4.964,5.188,5.841,3.825$ and 2.269$) \mathrm{kg} \mathrm{NH3Ha-1}$ respectively . At last the results showed a significantly differences at active ammonia volatilization at levels probably (0.01) . 
بمنخل سعة فتحاته ب ملم بعد عملية طحنه وقدرت بعض الصفات المقدمةة

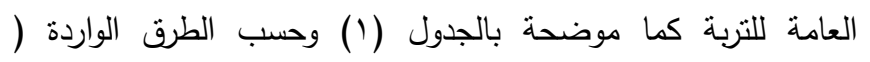

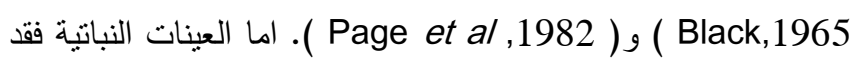
جمعت من محافظة النباتية واجرى عليها الاتي :-

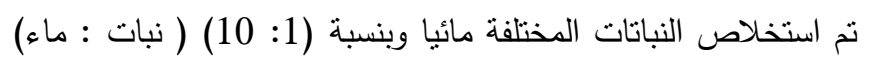

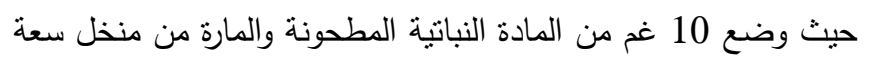

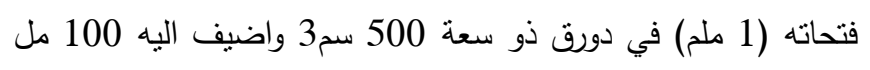
ماء مقطر ورج المزيج في رجاج ميكانيكي (Mechanical shaker)

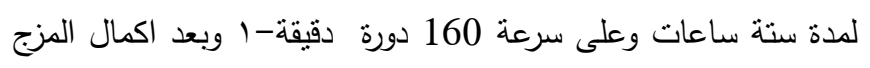

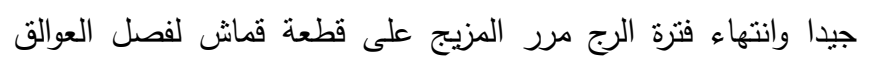

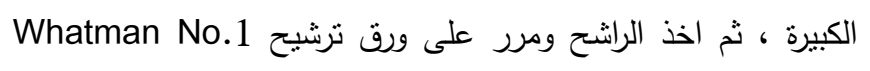
وذلك لغرض الحصول على محلول رائق، وقد جمع المحلول الرائق تُّن

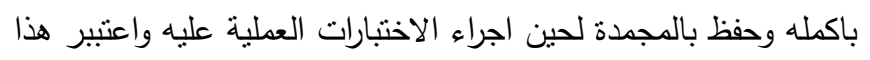
المحلول هو المحلول الاصلي للاجزاء النباتية المدروسة.

\begin{tabular}{|c|c|c|}
\hline \multicolumn{3}{|c|}{ جدول (1) الخصائص الكيميائية والفيزيائية للتربة المدروسة } \\
\hline القيمة المحسوبة & وحدة القياس & الصفة \\
\hline 7.49 & & $\mathrm{pH}$ \\
\hline 2.3 & $\mathrm{dS} \mathrm{m}-1$ & Electrical conductivity \\
\hline 30 & $\mathrm{mg} \mathrm{kg}-1$ & $\mathrm{CaCO} 3$ \\
\hline 16.6 & $\mathrm{~g} \mathrm{~kg}-1$ & TOC \\
\hline 28.6 & $\mathrm{~g} \mathrm{~kg}-1$ & O.M \\
\hline 1.42 & $\mathrm{~g} \mathrm{~kg}-1$ & Total nitrogen \\
\hline 11.60 & & $C: N$ ratio \\
\hline 6.5 & $\mathrm{mg} \mathrm{kg}-1$ & $\mathrm{NH} 4+$ \\
\hline 120 & $\mathrm{mg} \mathrm{L}-1$ & $\mathrm{Ca}+2$ \\
\hline 84 & mg L-1 & $M g+2$ \\
\hline 80 & $\mathrm{mg} \mathrm{L}-1$ & $\mathrm{Na}+$ \\
\hline 78.55 & $\mathrm{mg} \mathrm{L}-1$ & $\mathrm{~K}+$ \\
\hline 12.3 & mg L-1 & NO3_ \\
\hline 0 & $\mathrm{mg} \mathrm{L}-1$ & NO2- \\
\hline 195 & $\mathrm{mg} \mathrm{L}-1$ & $\mathrm{HCO}-3$ \\
\hline 290 & $\mathrm{mg} \mathrm{L}-1$ & SO4-2 \\
\hline r9o & $\mathrm{mg} \mathrm{L}-1$ & $\mathrm{Cl}-$ \\
\hline 30.5 & CCmole /100 gm soil & CEC \\
\hline 328 & \multirow{3}{*}{$\mathrm{g} \mathrm{kg}-1$} & Sand \\
\hline 196 & & Slit \\
\hline 476 & & Clay \\
\hline Clay & & Texture \\
\hline
\end{tabular}

يعد اليوريا من الاسمدة لرئيسية المستخدمة بنطاق واسع في العالم

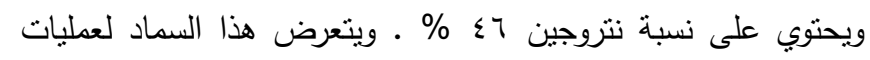
الفقد كالنطاير نتيجة التحلل المائي التي تحدث ندئ بواسطة انزيم اليوريز

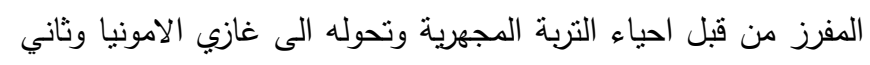

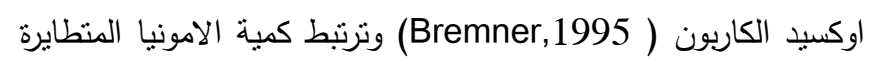

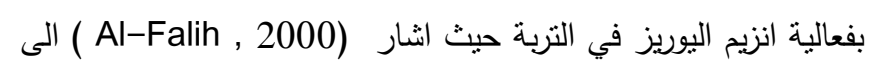

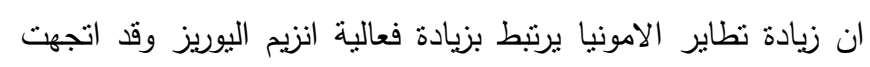

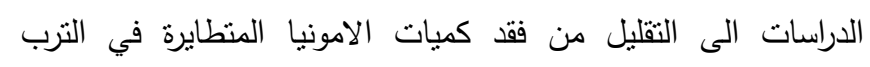
باستخدام المركبات الكيمباوية المختلفة كمواد مثبطة لانزيم اليوريز

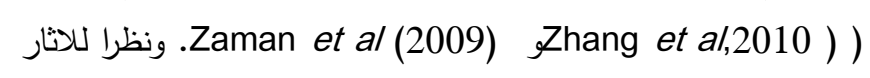
السيئة لبعض المركبات الكيمياوية المثبطة لانزيم اليوريز قد اثرت على

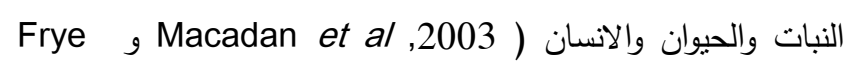
2005, ) • لذا التجأ بعض الباحثنين لاستخدام مواد اكثر امنا على وانى

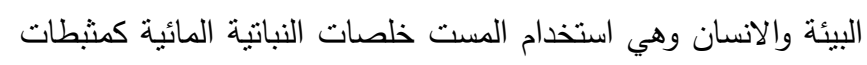

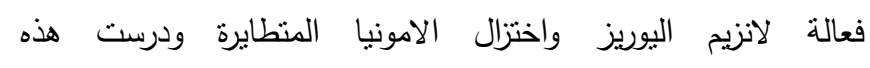

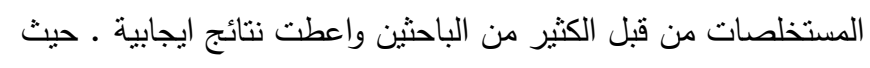

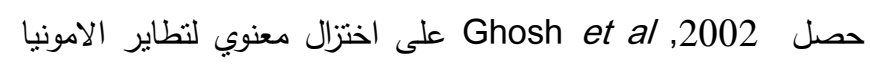

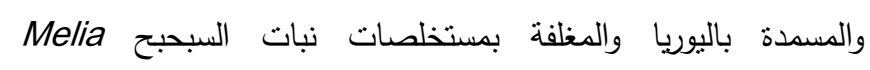
azdarach واوراق الصنوبر Pinus rexhurghi بعد تثبيط انزيم

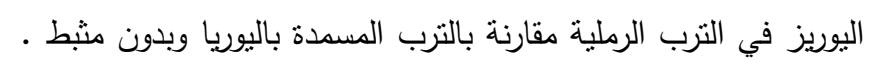

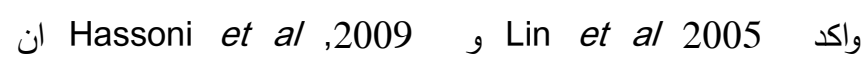
المستخلصات النباتية المائية ثبطت فعالية انزيم اليوريز بشكل ملحوظ

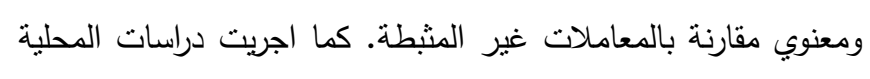

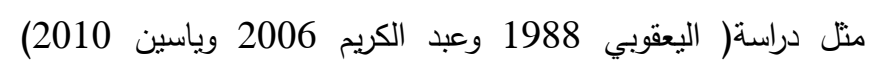
لمعرفة دور المستخلصات النباتية المائية في اختزال كميات الامونيا

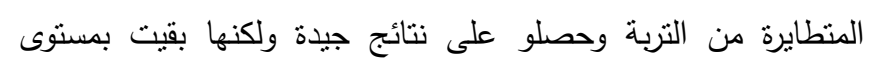

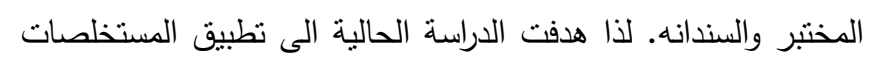

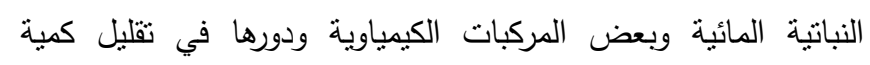
الامونيا حقليا وبنطاق تطبيقي اوسع على نبات الذرة البيضاء في اهوار النياء

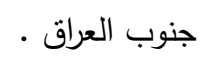

\section{المواد وطرق العمل}

اجريت التجربة الحقلية لترب اهوارجنوب العراق (هور ابو زرق )

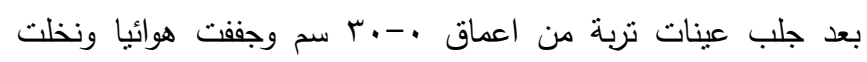


الهيدركينون Hydroquenione و ثايو كبريتات الامونيوم (ATS) Ammonium Thio Sulfate المثبطات بمعدل · 1 \% من وزن اليوريا المستخدمة .

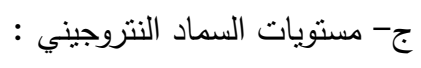
ويتضمن هذا العامل استعمال السماد النتروجيني وبواقع (100 ،

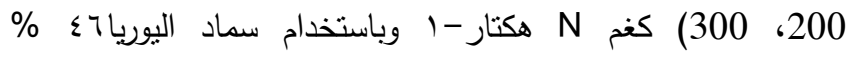

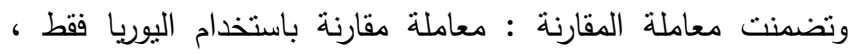

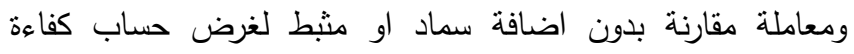
استعمال السماد. واجريت مكافحة للحقل لمعالجة الاصابات الحشرية بين السيقان وابط الاوراق وذللك باستعمال مبيد الدايزنون المحبب لمرتين

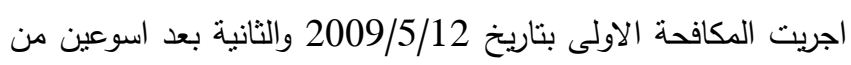
الاولى وبتاريخ 2009/5/27. ومن التجربة الحقلية نم تقدير كمية الامونيا المنطايرة :

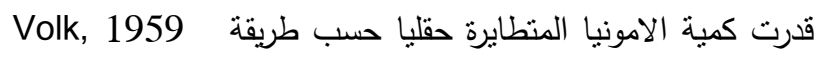
المعتمدة على استخدام حامض boric aid 2 o 2 لاقتتاص الامونيا المنطايرة مع اجراء بعض التحويرات حيث وضع 20 مل من حامض فل 20 boric aid 2 \% في اناء زجاجي والذي يحوي على خليط دليل بحرئ Bromo Cresol Green الصوف الزجاجي المتبع لمسك الامونيا المنطايرة وقد غطيت الاواني

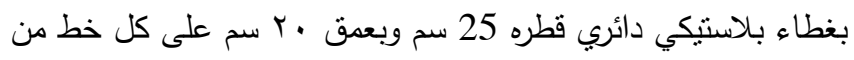

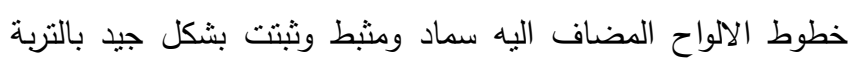
لضمان عدم تسرب جزء من غاز الامونيا المنطايرة ولفترات الزمنية

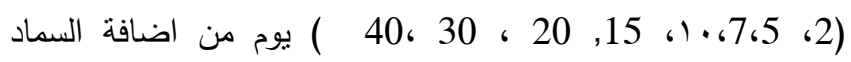

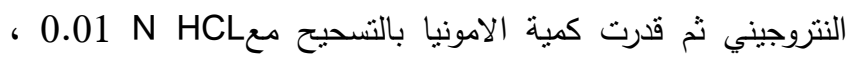
وحسبت كمية الامونيا المنطايرة بالملغم وفق القانون التالي :

$$
\begin{aligned}
& \text { : NH3/ Area = N *V * } 14 \\
& \text { HCL عبارية = N } \\
& \text { =V }
\end{aligned}
$$

وصمدت التجربة بالتصميم العشوائي للتجربة العاملية وفقا للراوي وخلف التفي

الله (1980)

\section{النتائجج والمناقشة:}

يوضح الثكل (1) تأثير معاملة اليوريا بالمستخلصات النباتيه

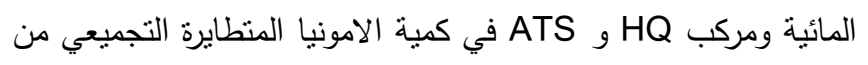

\begin{tabular}{|c|c|c|c|}
\hline تاريخ اخذ & الجزء الددروس & الاسم العلمي & اسم النبات \\
\hline $\begin{array}{c}30 \\
\text { شباط } 2008\end{array}$ & ورق & $\begin{array}{c}\text { Eucalyptus } \\
\text { camaldulensrs } \\
\text { Dehnl }\end{array}$ & يوكاليبتوس \\
\hline $\begin{array}{c}30 \\
\text { شباط } 2008\end{array}$ & ورق & $\begin{array}{c}\text { Myrtus communis } \\
\text { L. }\end{array}$ & الياس \\
\hline $\begin{array}{l}2 \\
2009\end{array}$ & جذور & $\begin{array}{c}\text { Cynodon dactylon } \\
\text { L.Pers. }\end{array}$ & الثيل \\
\hline $\begin{array}{c}30 \\
\text { الاول كانون } 2008\end{array}$ & ليف النخيل & $\begin{array}{c}\text { Phoenix dactlifera } \\
\text { L. }\end{array}$ & نخيل التمر \\
\hline $\begin{array}{c}15 \\
\text { كانون } 2008 \text { الثاني }\end{array}$ & ورق وسيقان & Oryza sativa & الرز \\
\hline
\end{tabular}
التربة بعد 40 يوما من الزراعة ويلاحظ ان معاملة سماد اليوريا بهذه الئه
جدول (2) يوضح جزء النبات الددروس

\section{التجرية الحقلية}

تم اجراء حراثتين متعامدنين في منتصف شهر اذار /2009 وقسمت الى قطع مسنطبلة بابعاد (3×4) منر ووزعت القنوات الحقلية بشكل يؤمن ري منتظم للالواح ، وزرعت بذور الذرة البيضاء بتاريخ

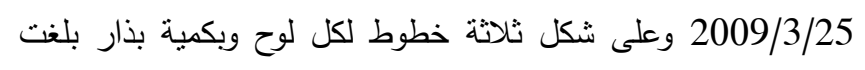

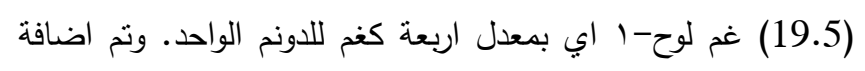

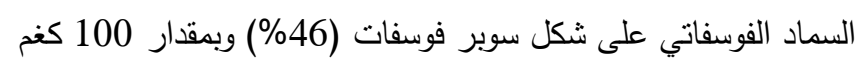

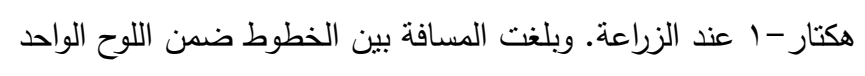

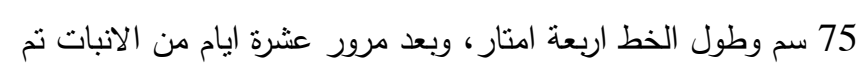

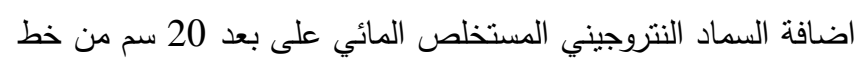
النبات وبعمق 5 سم في التربة واستخدت منبطات لانزيم اليوريز وتضمن هذا العامل 1- استعمال المثبطات التالية :1 - المستخلصات النباتية المائية : وشملت مستخلصات التمات اوراق الياس

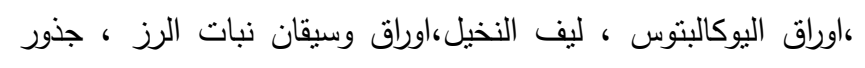

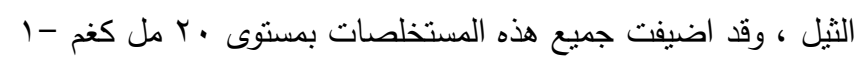

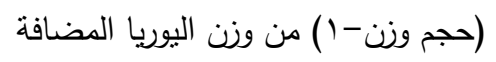
ب- المثبطات الكيمياوية : بن وزن التونات 
استخدام مستخلصات نبات السبحبح Melia azadarach واوراق نبات الصنوبر Pinus rexhurghii ادى الى تثنيط فعالية انزيم اليوريز في الترب الرملية الملحية واختزال تطاير الامونيا في الترب. اشنار Krajewska (2009) الى ان تغليف البوليمرات بالعناصر النادرة

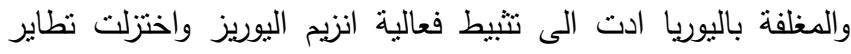
الامونيا مقارنة بفعالية انزيم اليوريز المضاف للترب المعاملة بسماد

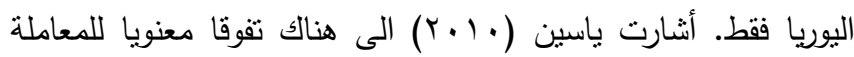
المضاف لها مستخلص مائي لنبات رز العنبر وبمستوى احتمالية ه..

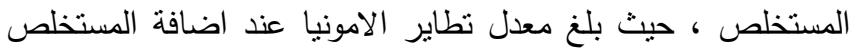

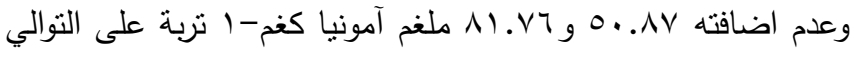
واعزت السبب في ذلك الى ان المستخلص قد ثبط انزيم اليوريز وعرقل تحلل اليوريا مائيا وعمل على اختزال نطاير الامونيا. وتتفق نتائج

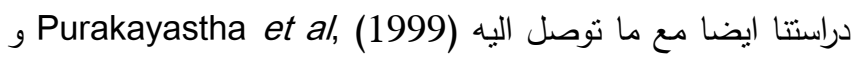
Hassoni et al, (2009) و الذي استخدموا Lin et al,(2005) مستخلصات نباتية ادت الى تثتيط انزيم اليوريز والتقليل من نطاير الامونيا وبالتالي ادت الى تحسين كفاءة الوحدة السمادية ومنهم.

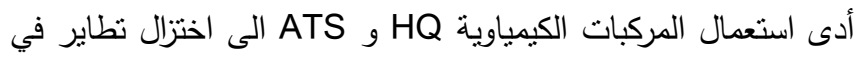
كمية الامونيا المنطايرة من التربة وبصورة معنوية باحتمالية 0.01

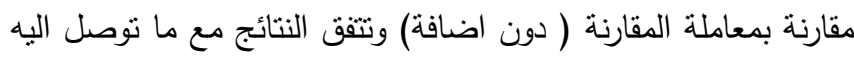
الذين Patra et al, (2009) و من (2003) لاحظوا ان اضافة مركب DCD مع اليوريا خفض من كمية الامونيا

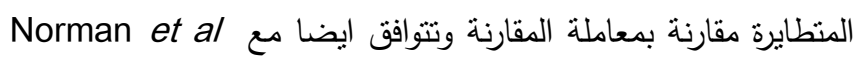

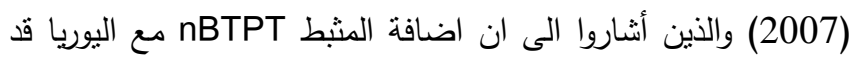

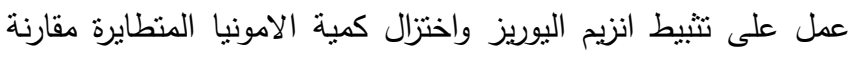

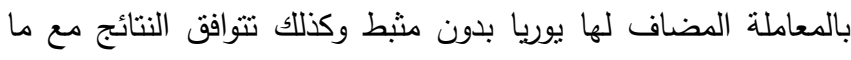

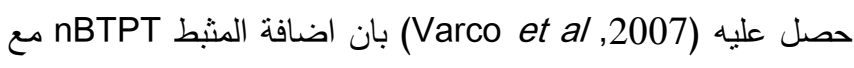
الأسددة النتروجينية في الترب غير المحروثة قد سبب اختزال نطاير الامونيا وحسن من إنتاجية القطن المزروعة في تلك الترب. ويوضح الثكل (1) تفوق بعض المستخلصات النباتية المائية في اختزال نطاير الامونيا وتثبيط فعالية انزيم اليوريز على المركبات الكيمياوية HQ و ATS وقد يعود السبب في ذللك الى احتواء المستخلصات النباتية المائية على عدد من المركبات الثانوية الايضية

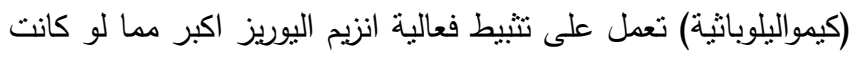

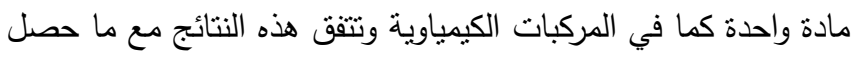

المواد آدت الى انخفاض في كمية الامونيا المنطايرة مقارنة بمعاملة

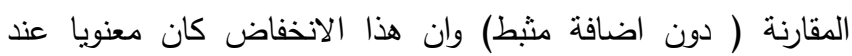
مستوى احتمالي 0.01 لجميع المعاملات مقارنة بمعاملة دون اضافة الفان.

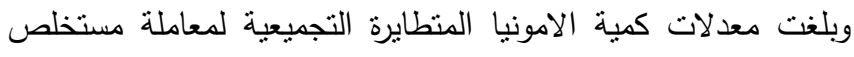

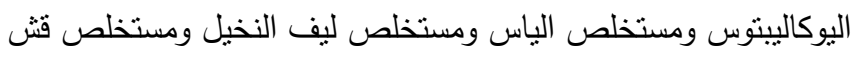
الرز ومستخلص جذور الثيل ومركب HQ و ATS (30.320 و

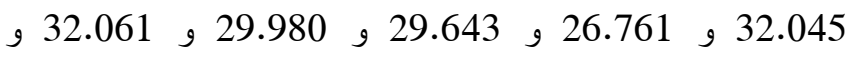
31.165 )كغ NH3 هكتار - 1 مقارنة بمعاملة المقارنة (بدون اضافة) والتي بلغت 36.633كغ مH3 هكتار -1 . . اختلفت معاملات المستخلصات النباتية المائية معنويا وبمسنوى احتمالية 0.01 فيما بينها كما تفوقت معاملات المستخلصات النباتية المائية في خفض كمية

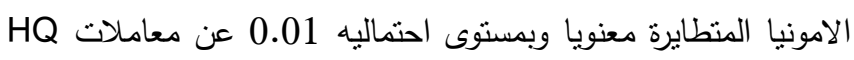

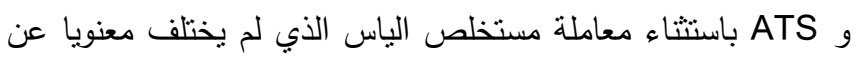

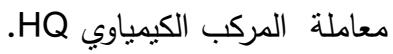

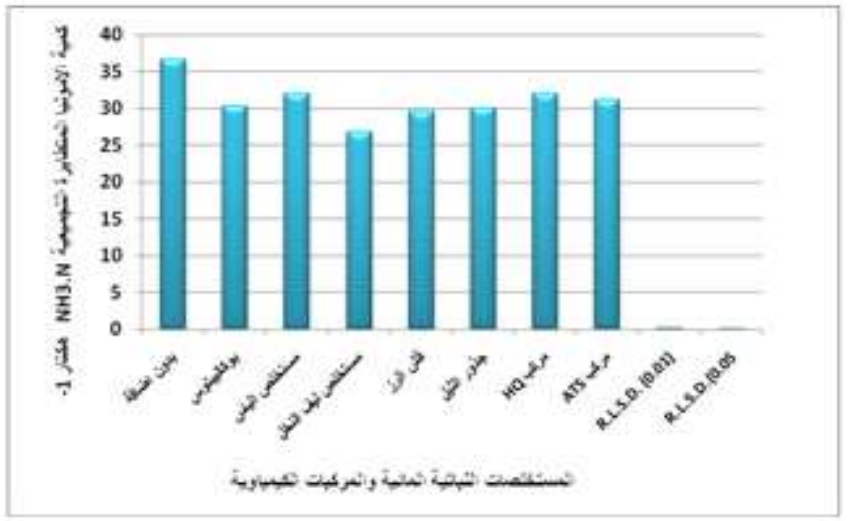

الثكل (1) تأثير أنواع المستخلصات النباتية المائية والمركبات الكيمياوية في كمية الامونيا المنطايرة التجميعية كغم NH3-N3

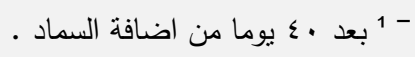

وقد يعود سبب اختلاف المستخلصات المائية النباتية فيما بينها في

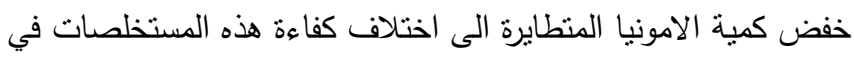

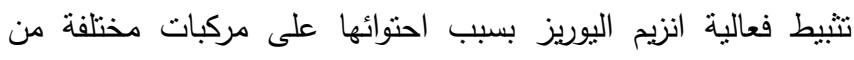

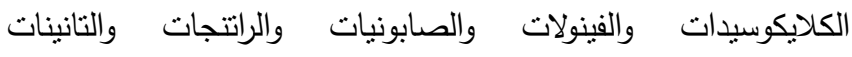
والكورمانيات ... وغيرها ) وتتفق هذه النتائج مع ما اشار اليه عبد وليدات الكريم (2006) الذي وجد اختلاف في كفاءة المستخلصات النباتية المائية في خفض كمية الامونيا المنطايرة من سماد اليوريا ـ وتنوافق

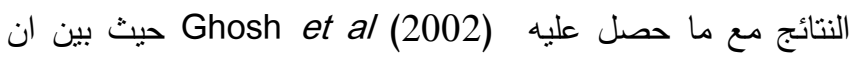


المتطايرة الفعلية بين الفترات جميعها معنويا وبمستوى احتمالية 0.01،

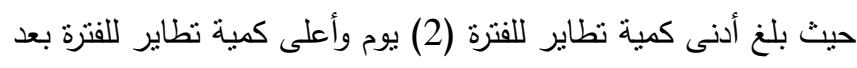

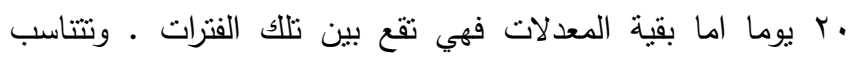

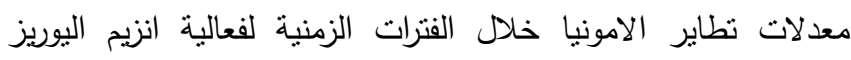

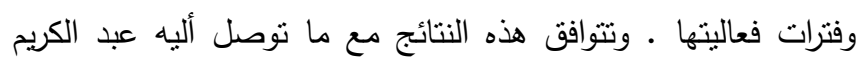

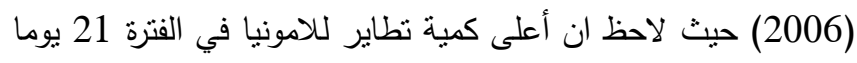
واقلها عند الفترة يوم واحد وللاستفادة العملية من تقليل نطاير الامونيا

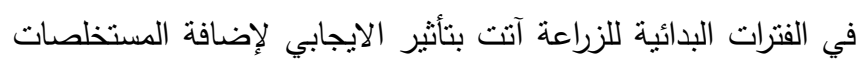

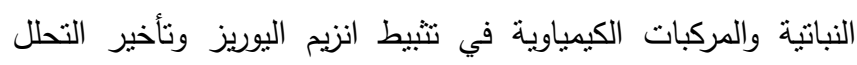

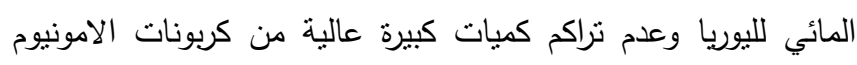
وتتجلى الأهمية التطبيقية لهذه النتيجة الى زيادة فرصة استفادة النبات من النتروجين المضاف بشكل سماد يوريا خلال الفترات الأولى للزراعة.

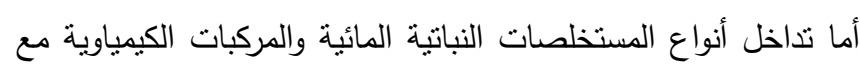

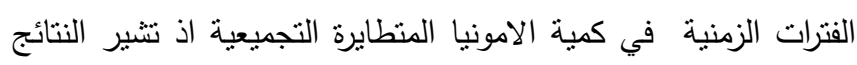
في جدول (1) أدناه تثير الى ان كمية الامونيا المنطايرة عند معاملة

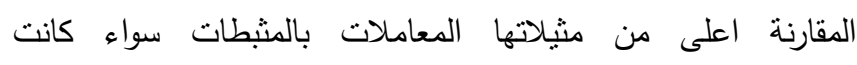

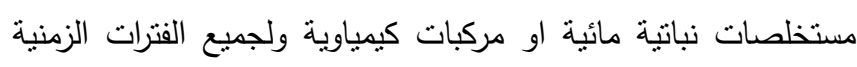

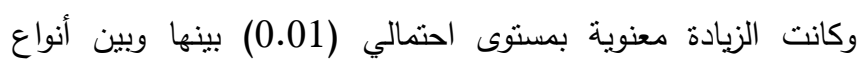
المستخلصات النباتية والمركبات الكيمياوية عند نفس الفترة

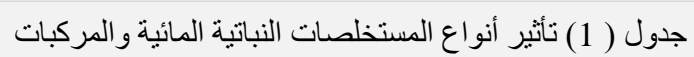

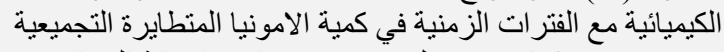

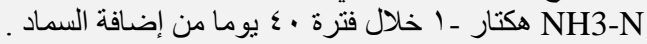

\begin{tabular}{|c|c|c|c|c|c|c|c|c|}
\hline \multicolumn{8}{|c|}{ Lل } & \multirow[b]{2}{*}{ العيلت } \\
\hline 40 & 30 & 20 & 15 & 10 & 7 & 5 & 2 & \\
\hline 36.633 & $33.14^{-}$ & 28.834 & 22524 & 16882 & 11.522 & 70582 & 3376 & - برد إنقية \\
\hline 3032 & 27.605 & 23.911 & 18.411 & 13.442 & 8.359 & 5.244 & 2234 & 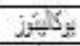 \\
\hline 32.045 & 29,050 & 25241 & 19310 & 13838 & 8376 & 5.130 & 2142 & 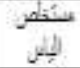 \\
\hline 26.761 & 24.675 & 21.222 & $15.74 !$ & 10.768 & 6.717 & 4.45 & 1809 & 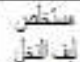 \\
\hline 29.643 & 26.926 & 22960 & 17.069 & 12.688 & 7.561 & 4.850 & 2206 & jof \\
\hline 29.980 & 27381 & 23.819 & 17986 & 12959 & 8247 & 5.301 & 2277 & جوز \\
\hline 32.061 & 29.062 & 24.951 & 19229 & 14.145 & 8.997 & 5.788 & 254 & HQ \\
\hline 31.165 & 28.609 & 24916 & 18861 & 13582 & 8541 & 5.603 & 2375 & $\begin{array}{l}\text { A } \\
\text { ATS }\end{array}$ \\
\hline
\end{tabular}

R.LS.DT(TYP) $0.05=0.368 \quad 0.01=0.472$

ويوضح الثكل (T) والتحليل الإحصائي في ملحق (Y) وجود تأثيرا معنويا لمستويات اضافة النتروجين في كمية الامونيا المنطايرة التجميعية من التربة إذ يلاحظ من الثكل ان زيادة كمية النتروجين
عليه عبد الكريم (2006) والذي أوضح كفاءة مستخلص الياس والليف وتفوقهما المعنوي في خفض فعالية انزيم اليوريز واختزال تطاير الامونيا

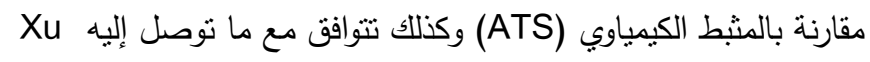

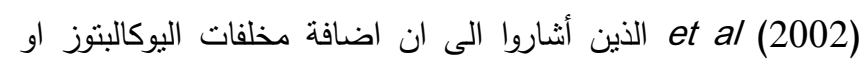

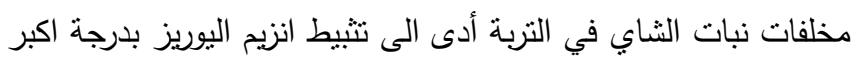

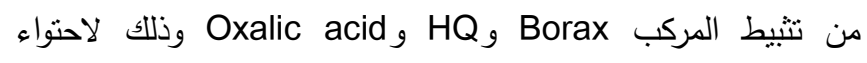

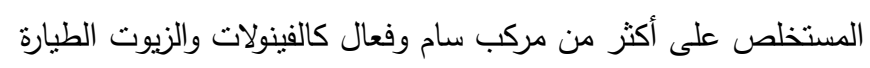

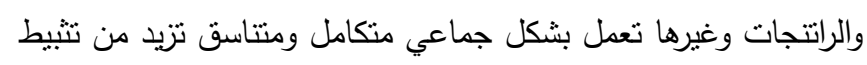

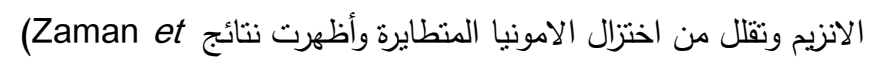

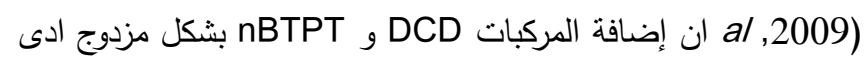

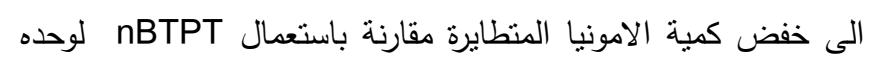

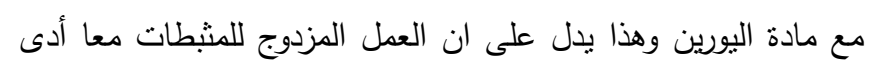

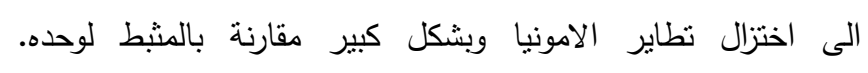

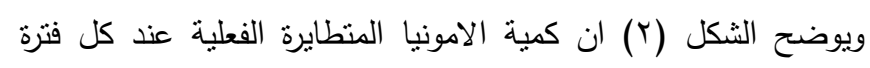

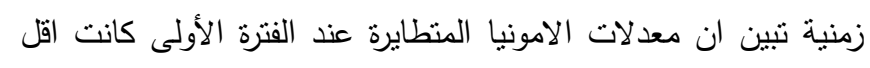

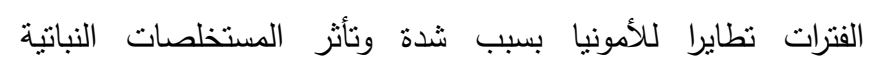
والمركبات الكيمياوية في بداية الإضافة وازدادت كمية الامونيا المنطايرة معنوية للفترات التي بعدها حتى وصلت الى أعلى قيم لكمية الامونيا المتطايرة الفعلية عند 20 يوما من الإضافة.

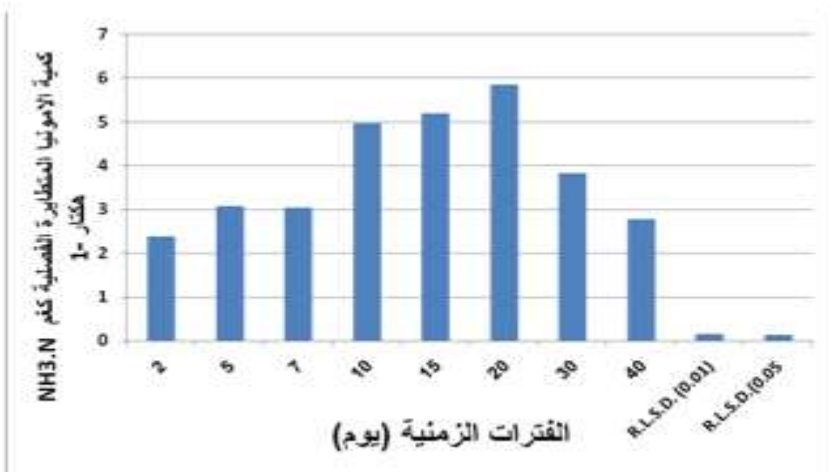

الثكل (r) تأثثر الفترات الزمنية المختلفة في كمية الامونيا المتطايرة الفعلية كغم NH3-N هكتار - 1 بعد فترة 40 يوماً من اضافة السماد.

وبلغت معدلات كمية الامونيا المنطايرة الفعلية للفترات الزمنية (2 و 5 (20)

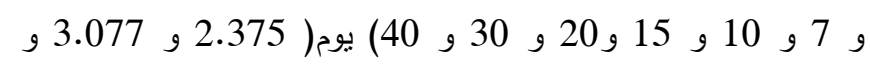

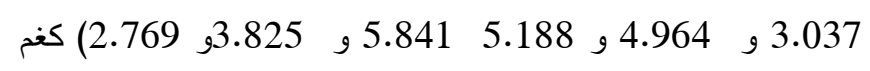
هكتار - 1 على النوالي. وكان الفارق في كمية الامونيا 
وكان للتناخل بين أنواع المستخلصات النباتية المائية والمركبات

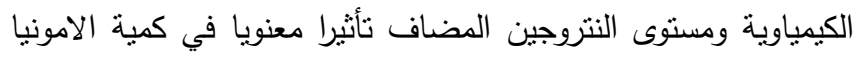
الكلية المفقودة (جدول 4) حيث حصل أعلى فقد للامونيا عند المستوى دئي النتروجيني 300 كغ نتروجين هكتار -1 وعند جميع انواع المنبطات المدروسة يليها في ذللك المستوى النتروجيني 200 كغم نتروجين هكتار -1 اما المستوى النتروجيني 100 كغم نتروجين هكتار -1 فأعطى اقل كميات تطاير ولجميع المعاملات ، حصل اقل كمية نطاير للامونيا المستخلصات اليوكالييتوس والياس وليف النخيل عند المستوى

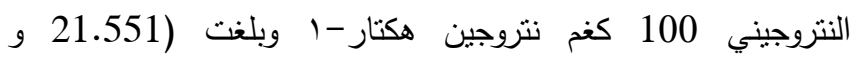
21.701 و 21.584 )كغ NH3 هكتار - 1 على التوالي وبدون فرق معنوي فيما بينها ـ وقد بدت هذه المستخلصات النباتية فروقا معنوية

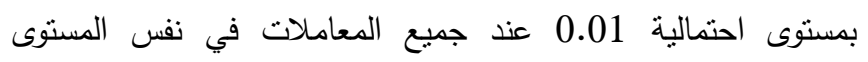
النتروجيني والمستويات النتروجينية الأخرى وقد حصل أعلى كمية

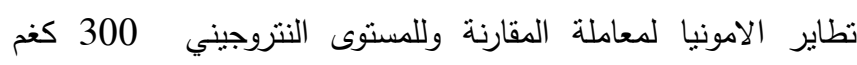

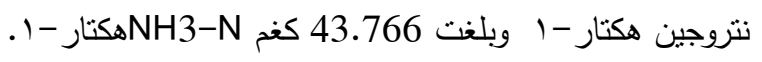

الجدول (ع ) تأثير تداخل انواع المستخلصات المائية للنبات و المركبات

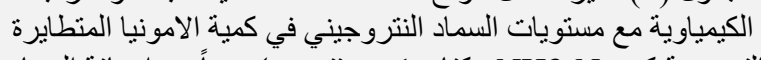

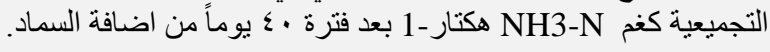

\begin{tabular}{|c|c|c|c|}
\hline \multicolumn{3}{|c|}{ N مستويات السماد النتروجيني كغم } & \multirow[t]{2}{*}{ المعاملات } \\
\hline 300 & 200 & 100 & \\
\hline 43.766 & 37.221 & 28.912 & بدون اضافه \\
\hline 38.316 & 31.103 & 21.551 & مستخلص اليوكاليتوس \\
\hline 40.989 & 33.446 & 21.701 & مستخلص الياس \\
\hline 31.860 & 26.840 & 21.584 & مستخلص ليف النخيل \\
\hline 29.643 & 29.534 & 25.648 & مستخلص قش الرز \\
\hline 34.419 & 30.873 & 24.648 & مستخلص جذور النخيل \\
\hline 37.214 & 32.081 & 26.889 & مركب HQ \\
\hline 36.289 & 32.262 & 24.944 & مركب ATS \\
\hline
\end{tabular}

R.L.S.D (T×N) O.O5=0.2260.01=0.290

أما تأثثر التداخل للفترات الزمنية ومسنويات السماد النتروجيني في كمية

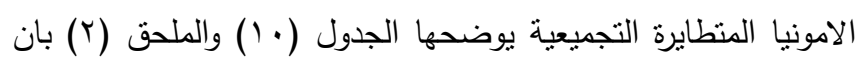
كمية الامونيا المنطايرة التجميعية تزداد بزيادة مستوى السماد النتروجيني

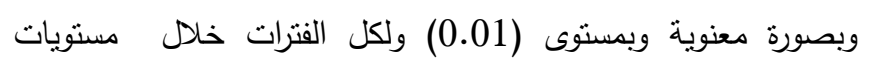

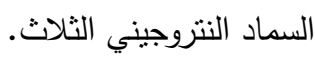

المضافة من 100 كغم نتروجين هكتار -1 الى 200 كغم نتروجين

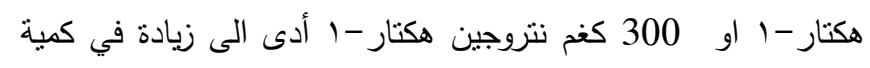
الامونيا المنطايرة التجميعية من التربة خلال 40 يوم حيث بلغت لهنت

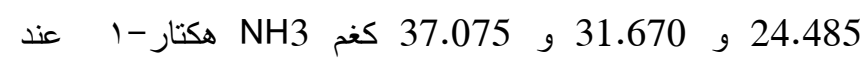
المستويات أعلاه على التوالي وبفرق معنوي بمسنوى احتمالية (0.01) تتفق هذه النتائج مع ما حصل عليه عبد الكريم (2006) الذي الشار

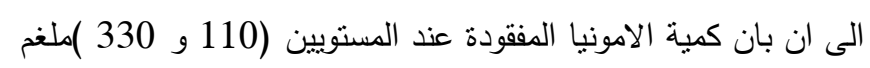

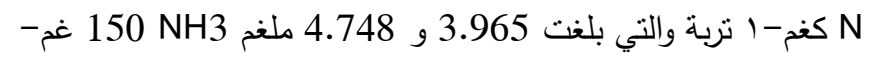

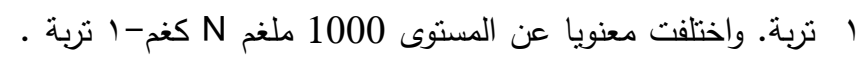

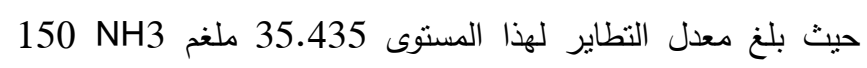

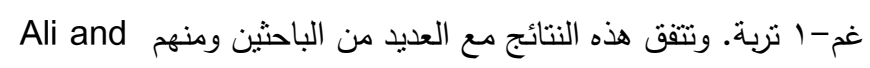
(1991) و ذو ذياب (1996) واليعقوبي (1988) الذين اثنتوا زيادة فقد الامونيا من السماد النتروجيني بزيادة مستويات السماد

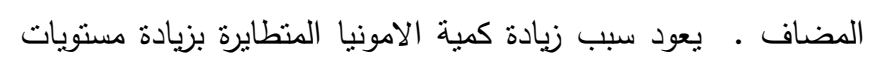

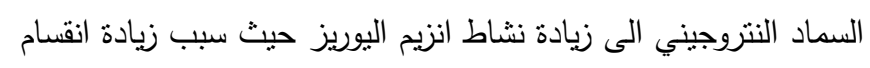

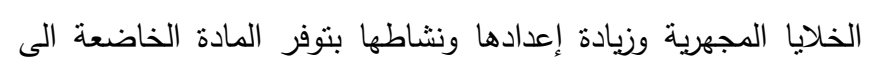

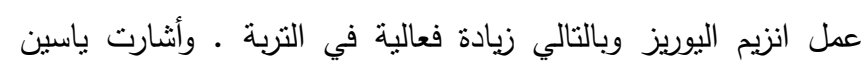

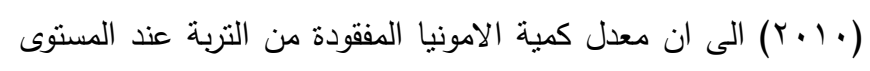

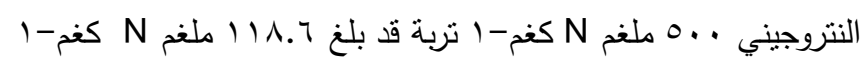

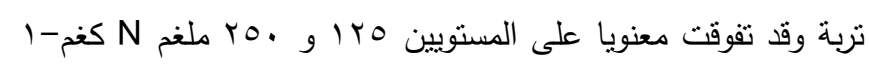

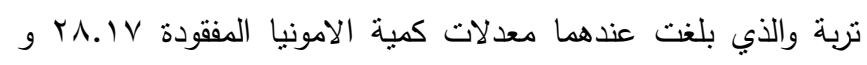

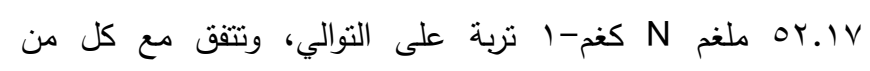
و Ma et al 2010 Hofman and VanCleemput (2005) . Alberto et al (2010) الامونيا من الحقول الزراعية يرتبط بزيادة كمية السماد النتروجيني

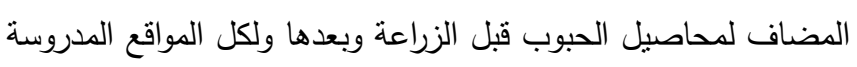

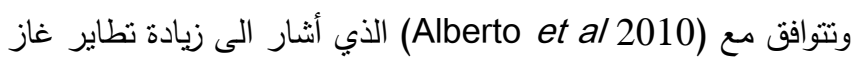
الامونيا بزيادة مستوى السماد المضاف بغض النظر عن مصدره .

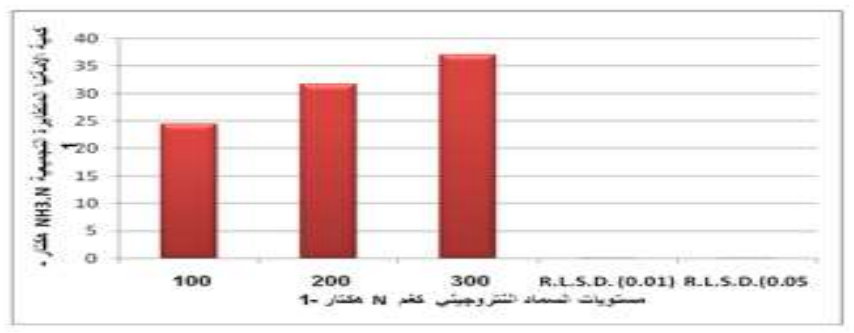

الثكل (†) نأثير مستويات السماد النتروجيني في كمية الامونيا المنطايرة

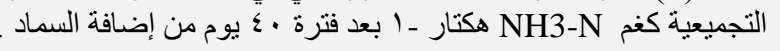




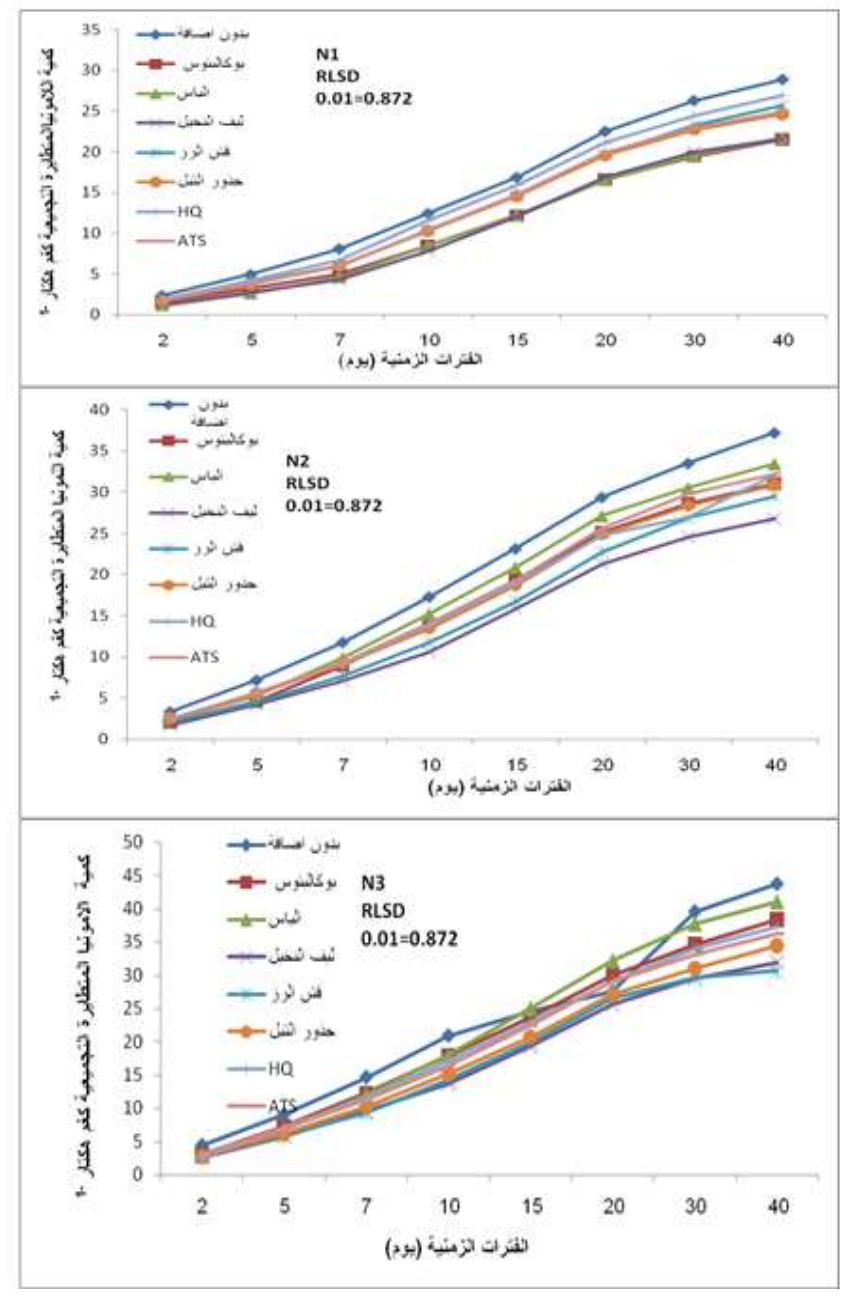

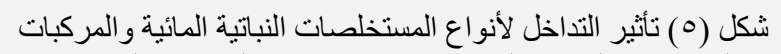

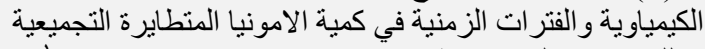

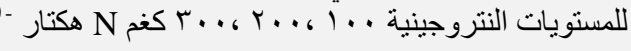

\section{References}

الانصساري، عبد المهدي صـالح ومحمد عبداله عبد الكريم ( • • ب).

دور المستخلصـات المائيـة لبعض النباتـات في خفض كميـة الامونيا المتطايرة من سماد اليوريا. المجلة الاردنيـة في العلوم

$$
\text { الزراعية. المجلد } 7 \text { العدد 1 : 1-1-9.9. }
$$

الراوي، خاشـع محمود وعبد العزيز محمد خلف الهه (•191 ) ). تصـيم وتحليل التجارب الزراعية. وزارة التعليم العالي والبحث العلمي،

$$
\text { جامعة الموصل، دار الكتب للطباعة والنشر • }
$$

اليعقوبي، كريم محسن حسن (9 (1) ). تثبيط نشاط أنزيم اليوريز في بعض الترب العراقية وتأثيره على نمو الذرة الصفراء والثـعير •

رسالة ماجسنير، كلية الزراعة، جامعة بغداد.
الجدول (0) تأثثر مستويات السماد النتروجيني مع الفترات الزمنية في كمية

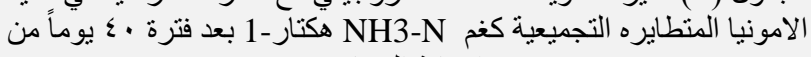

\begin{tabular}{|c|c|c|c|c|c|c|c|c|}
\hline \multicolumn{8}{|c|}{ 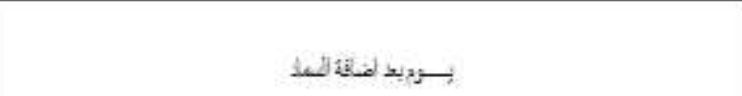 } & \multirow{2}{*}{ ستريت } \\
\hline 40 & 30 & 20 & 15 & 10 & 7 & 5 & 2 & \\
\hline 24.485 & 22314 & 19.086 & 14.125 & 9960 & 5.744 & 3.717 & 1.662 & 100 \\
\hline 31.670 & 28.915 & 25.137 & 19.126 & 13.789 & 9.170 & 5324 & 2.370 & 200 \\
\hline 37.075 & 33.692 & 29.222 & 22.674 & 16.611 & 11355 & 6810 & 3.094 & 300 \\
\hline
\end{tabular}
اضافة السماد.

كما يوضح الجدول (4) أن أعلى كمية لتطاير الامونيا التجميعية هو

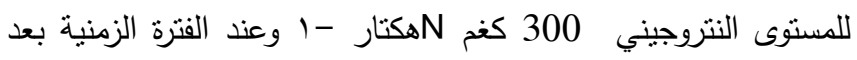
40 يوما وبلغت 37.075 كغم NH3 هكتار - 1 بينما بلغت اقل قيم

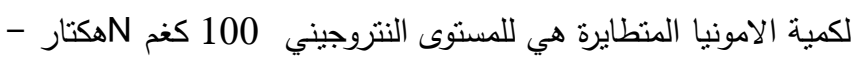

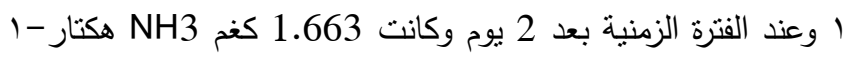
وكان الفرق معنوي للتداخلات وبمسنوى معنوي (0.01) ـ أما تأثنير

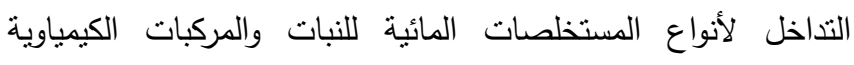
والفترات الزمنية في كمية الامونيا المتطايرة التجميعية للمستويات

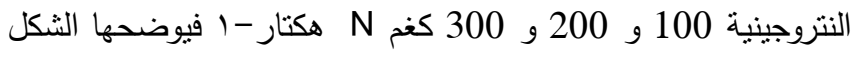
رقم (4) والذي يوضح تفوق جميع مستخلصات المائية للنبات والمركبات الكيمياوية على معاملة بدون اضافة ولجميع المسنويات

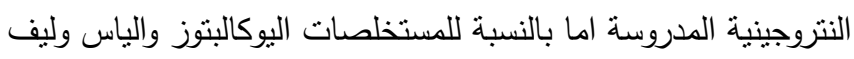

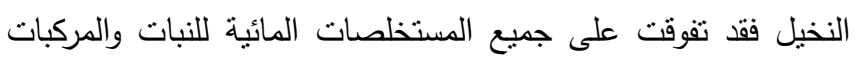
الكيمياوية الأخرى في خفض كمية الامونيا المنطايرة التجميعية عند

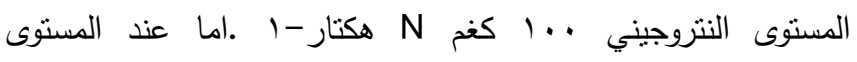

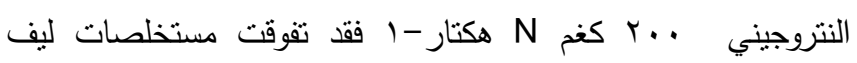
النخيل وجذور الثيل وقش الرز واليوكالبتوز معنويا على معاملة المقارنة

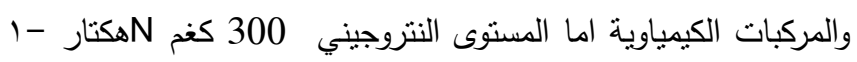
فقد لوحظ تفوق مستخلص ليف النخيل وقش الرز المائيين في خفض

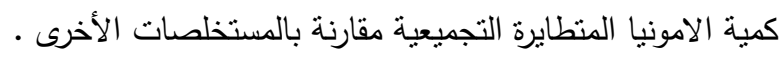


(Pinus roxburghi) and chinaberry (Melia azedrach) seed extracts on urea hydrolysis rate in a dandy soil. J. Indian Soc. Soil Sci. 50: 309311.

Ghosh ,B.N.;H.Chowdhury ; S.Kundu and H.S.Gupta (2002). Effect of pine needle (pinus roxburghi) and chinaberry Melia azedarach seed extracts on urea hydrolysis rate in a sandy soil.J.Indian Soc.Soil Sci.,50:309-311.

Hassoni, A. R., Ordouzadeh N., Ghaemi A.Amirmozafari N. Hamdi K,Nozari R.; (2009) Invitor inhibition of Helicobacter Pylori urease with non and semi fermented camellia sinensis .Indian .J.Med Microbiol.Jan.Mar.27(1):30-34.

Hofman, G. and O. VanCleemput (2005). Soil and plant nitrogen. Inter. Fertil. Indus. Asso Paris, France.robiol.Jan.Mar; 27(1) :30-34.

Kiran , U. and D.D. Patra (2003). Medicinal and aromatic plant materials as nitrification inhibitors for augmenting yield and nitrogen uptake of Japanese mint (Menthg arvensis L. Var. piperascens) . Biores . Tech. $86: 267-276$

Krajewska, B. (2009). Ureases. I. Functional, catalytic and Kinetic properties: A review. J. Molecular Cata. B: Enzymatic. 59: 9-21.

- Lin, Y.T.;Y.1.Kwon , R.G.Labbe and K.Shetty (2005) inhibition of Helicobacter Pylori and associated urease by Oregano and Graberry phyto chemical .

Ma. B. L; T. Y. Wu; N. Tremblay .W. Deen., N.B.Melaughlin.M.J,Morrison , and G.Stewart .(2010).On-Farm assessment of the amount and timing of Nitrogen fertilizeron Amona .Agroncmy ,j, V102 Issue I

Norman ,R.J.;T.L.Roberts ，T.W.Walker,C.E.Wilson .Jr.A.Enochs .D.I.Frizzell and I.D.B ranson (2007)Effect of Nurisphete on Ammonai -Volat ilization loss of urea and the grian yield of drillseeded,Delayed-flood Rice,B,R,Wells rice Researgh student(2007)AAES Reasearch Series 560.

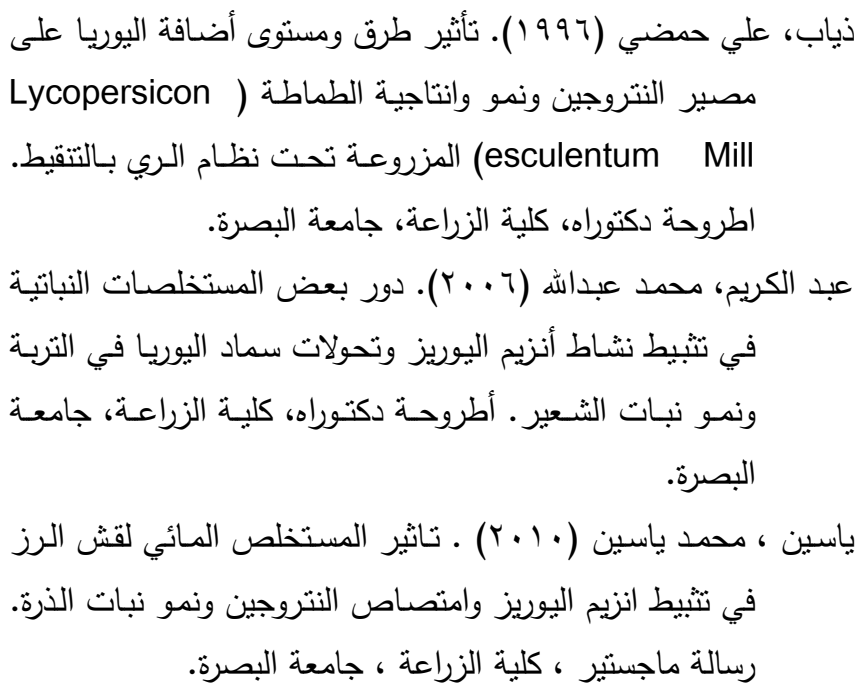

Al-berto,C, Campas Bernardi;Edson Pereira da Mota, Siumeire do Carmo Henriqu de Souza ;Rodrigo Donizeti Cardoso;Patricia Peronti Anchao Oliviera (2010) Ammonai volatilization ,dry matter yield and nitrogen levels of Italian ryegrass fertilized with urea and zeolite,2010 9 ${ }^{\text {th }}$ World Congress of soil science, soil solution for achanging World.1-6 August ,Brisban,Australia.

Al-Falih .Abdullah M. ;(2000). Urea hydrolysis and urease activity in Saudi Arabian soils .Qater University science .J.20: 95-103.

Ali, A.S. and J.L.Stroehlein (1991) Reactions of urea phosphate in calcareous and alkaline soils:1. Ammonia volatilization commun, soil sci.plant Anal.,22:1243-1256.

Black C.A. 1965 . Methods of soil analysis - part - II. Soc. Agron. Inc. Publ. Madision Wisconsin U.S.A.

Bremner, J.M. (1995). Recent research on problems in the use of urea as a nitrogen fertilizer. Fertil. Res. 42: 321-329.

Frye , W. (2005) Nitrification inhibition for Nitrogen efficiency and environment protection .IFA international Workshop on Enhanced .Efficiency fertilizers Frankfurt, Germany ; 2830 june.Ghosh, B.N.; H. Choodhury; S. Kunda and H.S. Gupta (2002). Effect pine needle 
Macadam, X. M-B.; Drado, A; Merino. P.; Estavillo, J. M.; Pinto M.; Gonzalez-Muria , C.;(2003) Dicy and iamide and 3.4.dimethuyl pyrazole phosphate decrease $\mathrm{N} 2 \mathrm{O}$ emissions from grass land but dicy and diamide produces deleterious effects in clover.Journal of plant physiology. 160:1517- 1523 .

Page, A.L.; R.H. Miller and D.R. Keeney (1982). Methods of soil analysis. Part 2. 2nd ed. ASA Inc. Madison, Wisconsin, U.S.A.

Patra, D.D.; U. Kiran; S. Chand and M. Anwar (2009). Use of urea coated with natural products to inhibit urea hydrolysis and nitrification in soil. Biol. Fertil. Soils US: 617-621.

Purakayastha , TJ.Katyal JC, Goswami et al (1997) Evaluation of some modified urea fertilizer applied to rice.Fer.News.42:53- 56.

Varco,J.Jae ;Robert E.Earnet;jimmyD.Johnson and Collier Ville (2007) Ureaase inhibitor (Agrotiain ) effects on use efficiency in no-till cotton .Belt wide cotton conference NEW Orleans ,Louisiana Janury9-12.

Volk ,G.M.(1959). Volatile loss ammonia following surface application of urea to turforbare soil .Agron .J.51:746- 749 .

- Xu, W.; W.Z.Yin, J.Z. Yuan; and W.Juan mei (2002) use of several plant materials and chemicals to inhibit soil urease activity and increase nitrogen recovery rate of urea by plant.Pedosphere.12: 75-282.

Zaman, M.; Saggar ,J.D.Blennerhassett;J.Singh.(2009) Effect of urease and nitrification inhibitors on $\mathrm{N}$ transformation, gaseous emissions of ammonia and nitrous oxide, pasture yield and $\mathrm{N}$ uptake in grazed pasture system, soil Biology and Biochemistry 41:1270-1280.

Zhang ,L.Z.Wu, Y.Jiang , L.Chen , Y.Song , L.Wang ,J.Xie .Ma (2010) fate applied urea $15 \mathrm{~N}$ in a soil-maize system as a affected by urease inhibitor and nitrification inhibitor .Plant soil Environ.56,

(1):8-15. 